\title{
AVALIAÇÃO DAS PROPRIEDADES MECÂNICAS DE FIOS E BRAQUETES ORTODÔNTICOS ESTÉTICOS
}

\section{EVALUATION OF MECHANICAL PROPERTIES OF ORTHODONTIC ARCHWIRES AND BRACKETS}

\section{EVALUACIÓN DE LAS PROPIEDADES MECÁNICAS DE ALAMBRES Y BRACKETS DE ORTODONCIA ESTÉTICA}

\author{
Igor Geoffroy Barbosa Fagundes1; Guilherme Garcia Beani²; Monalisa Costa Mota2; Sandro Marchette;
} Liliana Ávila Maltagliati4; Murilo Matias ${ }^{4}$

Submetido: 08/08/2020

Aprovado: 03/11/2020

\section{RESUMO}

Objetivo: comparar as forças de deflexão nos fios ortodônticos estéticos em braquetes autoligáveis cerâmicos passivos e interativos. Métodos: Quatro corpos de prova foram montados em um modelo de simulação clínica e avaliados em uma Máquina de Ensaio Universal (Emic). Em uma placa de resina acrílica foram fixados 10 dispositivos, onde foram colados os braquetes, simulando o arco dentário superior em forma de parábola. $\mathrm{O}$ dispositivo de acrílico representante do incisivo central direito foi movimentado em ativações de 0 a $3 \mathrm{~mm}$, com velocidade constante de $0,5 \mathrm{~mm} / \mathrm{min}$. Testes de Análise de Variância e de Tukey, com significância de $5 \%(P<0,05)$ foram executados. Resultados: As maiores médias de força foram apresentadas pelos fios Aesthetic Polymer Aditek e Flexy NiTi Rhodium quando empregados com o braquete Clarity. Conclusão: Braquetes autoligáveis cerâmicos utilizados com diferentes fios de estéticos podem apresentar comportamentos diferentes devido às características dos materiais de recobrimento empregados nos fios.

DESCRITORES: Fios Ortodônticos; Braquetes Ortodônticos; Ortodontia

\section{ABSTRACT}

Objective: to compare the deflection forces on aesthetic orthodontic archwires in passive and interactive self-ligating ceramic brackets. Methods: Four specimens were assembled in a clinical simulation device and evaluated on a Universal Testing Machine (Emic). Ten devices were attached to an acrylic resin plate, where the brackets were attached, simulating the upper dental arch in the parabola form. The acrylic device representing the upper right central incisor was moved in activations of 0 to $3 \mathrm{~mm}$, with a constant speed of $0.5 \mathrm{~mm} / \mathrm{min}$. Analysis of Variance and Tukey tests, with a significance level of $5 \%(P<0.05)$ were performed. Results: The highest mean force was presented by the Aesthetic Polymer Aditek and Flexy NiTi Rhodium archwires when used with the Clarity bracket. Conclusion: Self-ligating ceramic brackets used with different aesthetic archwires may behave differently due to the characteristics of the covering materials used in the archwires.

KEYWORDS: Orthodontic Wires; Orthodontic Brackets; Orthodontics

\footnotetext{
${ }^{1}$ Aluno de graduação da Universidade Guarulhos - Centro, Curso de Odontologia, Guarulhos, SP. ORCID: 0000-0001-9965014X

${ }^{2}$ Cirurgião (a) dentista - Universidade Guarulhos (UNG) - Consultório particular. ORCID: 0000-0001-7047-2247; ORCID: 00000001-7918-1390

${ }^{3}$ Aluno pós-graduação da Universidade Guarulhos - Curso de Mestrado Profissional em Ortodontia, Guarulhos, SP. ORCID: 0000-0002-8036-2823

${ }^{4}$ Docentes de graduação e Pós-Graduação da Universidade Guarulhos - Centro, Curso de Mestrado Profissional em Ortodontia, Guarulhos, SP.ORCID: 0000-0003-4343-8048; ORCID: 0000-0002-0228-8920
} 


\section{RESUMEN}

Objetivo: comparar las fuerzas de deflexión sobre alambres de ortodoncia estética en brackets cerámicos de autoligado pasivo e interactivo. Métodos: Se ensamblaron cuatro muestras en un modelo de simulación clínica y se evaluaron en una máquina de prueba universal (Emic). Se colocaron 10 dispositivos en una placa de resina acrílica, donde se pegaron los brackets, simulando el arco dentario superior en forma de parábola. El dispositivo acrílico que representa el incisivo central derecho se movió en activaciones de 0 a $3 \mathrm{~mm}$, con una velocidad constante de 0,5 $\mathrm{mm} / \mathrm{min}$.. Se realizaron análisis de varianza y pruebas de Tukey, con una significancia del $5 \%(P<0.05)$. Resultados: Las resistencias medias más altas fueron presentadas por las roscas Aesthetic Polymer Aditek y Flexy NiTi Rhodium cuando se usaron con el soporte Clarity. Conclusión: Los brackets cerámicos autoligables utilizados con diferentes alambres estéticos pueden comportarse de manera diferente debido a las características de los materiales de recubrimiento utilizados en los alambres.

DESCRIPTORES: Alambres de Ortodoncia; Brackets de Ortodoncia; Ortodoncia 


\section{INTRODUÇÃO}

A mecânica ortodôntica é baseada no princípio da acumulação de energia elástica e transformação dessa energia em trabalho mecânico por meio da movimentação dos dentes. Cada ajuste do aparelho armazena e controla o mecanismo de transferência e distribuição das forças. Um ótimo controle do movimento dentário requer a aplicação de um sistema de forças específico, que é devidamente guiado por meio de acessórios, tais como os fios ortodônticos 1 .

Como o tratamento ortodôntico estende-se por vários meses, a aparência dos acessórios é avaliada pelos pacientes como um fator significativo a ser considerado. A demanda pela estética fez com que diversas empresas começassem a produzir, no final da década de 70 , braquetes não metálicos, de policarbonato ou cerâmicos. Atualmente, os braquetes estéticos representam uma realidade na clínica ortodôntica, oferecendo uma alternativa aos metálicos. Entretanto, o mesmo não ocorreu em relação aos fios estéticos, que foram pouco relatados na literatura ortodôntica até meados da primeira década do século XXI2.

Os braquetes autoligáveis, por sua vez, foram introduzidos em meados dos anos de 1930 na forma de acessório de Russel, e constituem sistemas de braquetes sem ligadura que apresentam um dispositivo mecânico que permite esta ligação por meio de abertura e fechamento da parede anterior do braquete3.

Com relação aos fios, seus materiais de recobrimento devem ter como requisitos a facilidade de aplicação em camadas finas, a resistência e o baixo coeficiente de friç̧ão, além da necessidade de serem biocompatíveis e possuírem aspecto estético agradável, compatível com a translucidez dos braquetes estéticos e com a coloração dos dentes. As empresas de materiais ortodônticos ainda investem na busca da cobertura ideal para os fios metálicos ortodônticos, a fim de torná-los estéticos e, ao mesmo tempo, eficientes mecanicamente. Os diferentes tipos de cobertura alteram algumas propriedades dos fios, tais como atrito e fricção4.

Assim, a justificativa do presente trabalho baseia-se nas poucas publicações científicas que são apresentadas até o presente momento na literatura, especificamente em relação aos fios estéticos e braquetes autoligáveis estéticos atuando simultaneamente, referentes às forças geradas pela deflexão de fios ortodônticos associados a diferentes tipos de braquetes autoligáveis.
O objetivo deste trabalho foi comparar as forças de deflexão geradas pelos fios ortodônticos de Níquel-Titânio (NiTi) com recobrimento estético, quando empregados em conjunto com braquetes autoligáveis cerâmicos passivos e interativos.

\section{MATERIAIS E MÉTODOS MATERIAL \\ Grupos experimentais}

Quatro modelos de simulação clínica foram utilizados neste estudo. Cada um deles recebeu um diferente tipo de braquete ortodôntico, variando de acordo com as características de abertura e fechamento do sistema, a saber: 2 modelos de braquetes estéticos autoligáveis passivos e 2 modelos de braquetes estéticos autoligáveis interativos.

Nesses modelos foram testados 4 diferentes fios de NiTi, de secção redonda de 0,016 ", todos com algum tipo de recobrimento estético, nos quais foram aplicadas forças para promover a sua deflexão de 0 até $3 \mathrm{~mm}$ durante a sua desativação. Foram 16 grupos, utilizando 7 fios por grupo, totalizando 160 testes. (Quadro 1).

Quadro 1: Discriminação dos materiais utilizados na pesquisa

\begin{tabular}{|c|c|c|c|c|c|}
\hline Fio & Calibre & $\begin{array}{c}\text { Marca } \\
\text { Comercial }\end{array}$ & Braquete & Tipo & $\begin{array}{c}\text { Marca } \\
\text { comercial }\end{array}$ \\
\hline \multirow{4}{*}{$\begin{array}{c}\mathrm{NiTi} \\
\text { com } \\
\text { revesti- } \\
\text { mento }\end{array}$} & \multirow{4}{*}{$0.016 "$} & $\begin{array}{c}\text { High Aesthetic } \\
\text { Nickel Titanium } \\
\text { Wire } \\
\text { (TP } \\
\text { Orthodontics) }\end{array}$ & \multirow{4}{*}{$\begin{array}{c}\text { Cerâmica } \\
\text { Policrista- } \\
\quad \text { lina }\end{array}$} & \multirow{2}{*}{$\begin{array}{l}\text { Autoli- } \\
\text { gável } \\
\text { passivo }\end{array}$} & $\begin{array}{l}\text { Damon } \\
\text { Clear } \\
\text { (Ormco) }\end{array}$ \\
\hline & & $\begin{array}{c}\text { Flexy NiTi } \\
\text { Rhodium } \\
\text { (Orthometric) }\end{array}$ & & & $\begin{array}{c}\text { Clarity } \\
\text { (Dentsply/ } \\
\text { GAC) }\end{array}$ \\
\hline & & $\begin{array}{l}\text { High Aesthetic } \\
\text { (GAC) }\end{array}$ & & \multirow{2}{*}{$\begin{array}{c}\text { Autoli- } \\
\text { gável } \\
\text { interativo }\end{array}$} & $\begin{array}{l}\text { QuicKlear } \\
\text { (Foresta- } \\
\text { dent) }\end{array}$ \\
\hline & & $\begin{array}{c}\text { Aesthetic } \\
\text { Polymer } \\
\text { (Aditek) }\end{array}$ & & & $\begin{array}{c}\text { In-Ovation } \\
\text { C } \\
\text { (Dentsply/ } \\
\text { GAC) }\end{array}$ \\
\hline
\end{tabular}

\section{MÉTODOS}

\section{Teste de deflexão}

Os dispositivos deste estudo foram afixados à placa de acrílico com uma canaleta respeitando uma distância padrão de $6 \mathrm{~mm}$ entre as aletas. $O$ formato da parábola foi determinado pela geometria do fio a ser testado, diminuindo o risco de geração de forças diversas das advindas por meio da deflexão aplicada para este estudo.

Para que a comparação das forças de deflexão ge- 
radas fosse fidedigna, os quatro modelos foram construídos de maneira idêntica e a aplicação de força foi realizada de maneira sistemática. Em cada dispositivo representante do incisivo central superior direito, de cada um dos quatro modelos, uma deflexão gradual de 0 a $3 \mathrm{~mm}$ foi aplicada. As cargas geradas foram analisadas em $0,5 \mathrm{~mm}, 1 \mathrm{~mm}, 2 \mathrm{~mm}$ e $3 \mathrm{~mm}$.

\section{Simulação clínica}

A Figura 1 representa o esquema do modelo de simulação clínica que foi utilizado neste estudo. Este modelo é composto de uma placa de resina acrílica preparada para receber os dispositivos de acrílico que representavam os dentes. Os braquetes, correspondentes a cada tipo de aparelho, foram colados com cianoacrilato em gel nos dispositivos e estes foram fixados por meio de parafusos rosqueados na parte de baixo da resina acrílica.

Figura 1: Modelo de simulação clínica com as estruturas em posição durante a execução dos testes de deflexão.

Os testes foram feitos na área correspondente ao

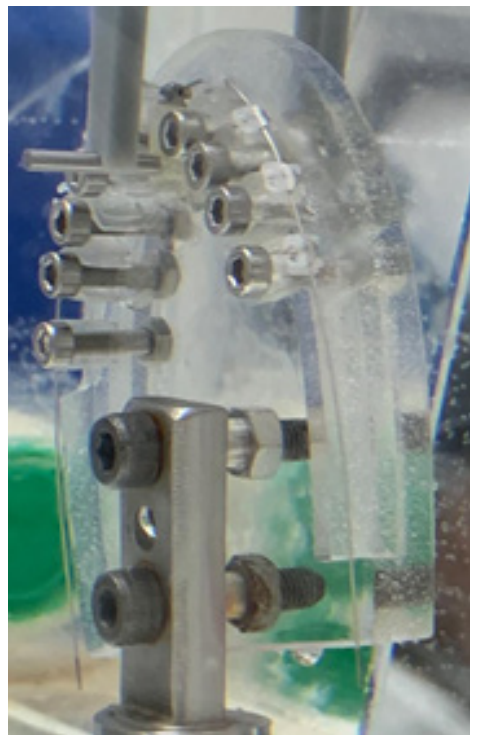

incisivo central. O cilindro correspondente a esse dente foi afixado ao arco e, posteriormente, desparafusado da placa de resina, para não alterar a distância inter-braquetes. A extremidade da ponta ativadora foi fixada na máquina de ensaios para promover o deslocamento. $\mathrm{O}$ modelo de simulação clínica foi fixado com o nivelamento adequado para que a linha de ação da força ativadora atuasse perpendicularmente ao plano do braquete.

A movimentação do braquete com o arco cor- responde clinicamente à ativação durante o aumento gradual da força e a desativação corresponde à diminuição gradual da força que provocou a deflexão. A velocidade da máquina de ensaio para o deslocamento, tanto na ativação quanto na desativação, foi de $0,5 \mathrm{~mm} / \mathrm{min}$. Foi utilizada uma máquina de ensaio EMIC com célula de carga de $50 \mathrm{~N}$, cuja precisão e sensibilidade possibilitaram resultados mais confiáveis.

\section{Análise estatística}

A distribuição normal das variáveis foi avaliada por meio do teste de Kolmogorov-Smirnov. Como todas as variáveis apresentaram uma distribuição normal, testes paramétricos, com média e desvio-padrão, foram empregados.

Após os testes de deflexão, os resultados das forças geradas foram avaliados por meio da análise de variância (ANOVA) a dois critérios de seleção, com nível de significância de 5\% para comparação dos grupos, seguidos pelo teste de Tukey nos casos em que apresentaram diferenças estatisticamente significantes $(p<0,05)$.

Todos os testes foram realizados com o auxílio do programa IBM® SPSS® Statistics, versão 23.

\section{RESULTADOS}

Os resultados das análises estatísticas realizadas na presente pesquisa estão descritos nas tabelas abaixo e mostram as comparações entre fios estéticos e braquetes autoligáveis. De maneira geral, foram observadas diferenças estatisticamente significantes em todas as deflexões estudadas $(0,5,1,2$ e $3 \mathrm{~mm})$.

A tabela 1 mostra a comparação dos diferentes tipos de braquetes quando empregados em conjunto com o fio Aesthetic Polymer Aditek. Os maiores níveis de força foram observados com o braquete Clarity, principalmente nas deflexões de $2 \mathrm{~mm}$ e $3 \mathrm{~mm}$.

Tabela 1: Média (em cN) e desvio padrão (D.P.) dos resultados do teste ANOVA a um critério de seleção e teste de Tukey, quando necessário, para avaliar o comportamento dos braquetes quando empregados com o fio Aesthetic Polymer Aditek ( $N=7)$ 


\begin{tabular}{|c|c|c|c|c|c|c|c|c|c|}
\hline \multirow[t]{2}{*}{ Variável } & \multicolumn{2}{|c|}{ Clarity } & \multicolumn{2}{|c|}{ Damon Clear } & \multicolumn{2}{|c|}{ In-Ovation } & \multicolumn{2}{|c|}{ Quicklear } & \multirow[t]{2}{*}{$\mathbf{P}$} \\
\hline & Média & D.P. & Média & D.P. & Média & D.P. & Média & D.P. & \\
\hline $3,0 \mathrm{~mm}$ & 4476,14 a & 583,64 & $\begin{array}{c}3606,78 \\
b, c\end{array}$ & 194,98 & $\begin{array}{c}4000,00 \\
a, c\end{array}$ & 425,82 & $\begin{array}{c}3776,33 \\
\text { b,c }\end{array}$ & 118,82 & $0,002^{*}$ \\
\hline $2,0 \mathrm{~mm}$ & 2112,55 a & 234,53 & $1817,46 \mathrm{~b}$ & 181,43 & $\begin{array}{c}1857,14 \\
\mathrm{a}, \mathrm{b}\end{array}$ & 169,29 & $\begin{array}{c}1866,52 \\
\mathrm{a}, \mathrm{b}\end{array}$ & 169,37 & $0,031^{*}$ \\
\hline $1,0 \mathrm{~mm}$ & 839,87 & 349,80 & 924,87 & 339,85 & 1035,74 & 527,00 & 987,73 & 250,71 & 0,792 \\
\hline $0,5 \mathrm{~mm}$ & 242,42 & 187,61 & 242,42 & 227,27 & 333,33 & 313,45 & 349,92 & 330,40 & 0,049 \\
\hline
\end{tabular}

${ }^{*}$ Estatisticamente significante para $p<0,05$.

Diferentes letras indicam a presença de diferença estatisticamente significante entre os grupos, indicados pelo Teste de Tukey.

A tabela 2 apresenta a comparação dos braquetes autoligáveis empregados com o fio Flexy NiTi Rhodium. Somente na deflexão de $2 \mathrm{~mm}$ foram encontradas diferenças estatisticamente significantes, novamente com as maiores forças sendo apresentadas pelo braquete Clarity.

Tabela 2: Média (em cN) e desvio padrão (D.P.) dos resultados do teste ANOVA a um critério de seleção e teste de Tukey, quando necessário, para avaliar o comportamento dos braquetes quando empregados com o fio Flexy NiTi Rhodium ( $\mathrm{N}=7$ )

\begin{tabular}{|c|c|c|c|c|c|c|c|c|c|}
\hline \multirow[t]{2}{*}{ Variável } & \multicolumn{2}{|c|}{ Clarity } & \multicolumn{2}{|c|}{ Damon Clear } & \multicolumn{2}{|c|}{ In-Ovation } & \multicolumn{2}{|c|}{ Quicklear } & \multirow[t]{2}{*}{$\mathbf{P}$} \\
\hline & Média & D.P. & Média & D.P. & Média & D.P. & Média & D.P. & \\
\hline $3,0 \mathrm{~mm}$ & 4035,04 & 192,24 & 3692,96 & 291,57 & 3800,81 & 436,50 & 3746,03 & 426,25 & 0,252 \\
\hline $2,0 \mathrm{~mm}$ & 2765,55 a & 458,40 & $1901,24 \mathrm{~b}$ & 144,13 & $\underset{c, d}{2361,31}$ & 110,62 & $\underset{b, c}{2022,00}$ & 219,05 & $0,001^{*}$ \\
\hline $1,0 \mathrm{~mm}$ & 1117,50 & 666,75 & 683,01 & 236,50 & 846,43 & 195,92 & 898,62 & 472,21 & 0,327 \\
\hline $0,5 \mathrm{~mm}$ & 250,18 & 244,36 & 188,94 & 126,99 & 167,05 & 90,99 & 312,77 & 243,16 & 0,494 \\
\hline
\end{tabular}

*Estatisticamente significante para $p<0,05$.

Diferentes letras indicam a presença de diferença estatisticamente significante entre os grupos, indicados pelo Teste de Tukey.

A tabela 3 apresenta a comparação dos braquetes autoligáveis estéticos com o fio High Aesthetic Nickel Titanium Wire. Novamente, o braquete Clarity apresentou as maiores médias de forças de deflexão, com valores estatisticamente significante para as deflexões de $1 \mathrm{~mm}$ e $2 \mathrm{~mm}$.

Tabela 3: Média (em cN) e desvio padrão (D.P.) dos resultados do teste ANOVA a um critério de seleção e teste de Tukey, quando necessário, para avaliar o comportamento dos braquetes quando empregados com o fio High Aesthetic Nickel Titanium Wire $(\mathrm{N}=7)$

\begin{tabular}{|c|c|c|c|c|c|c|c|c|c|}
\hline \multirow[t]{2}{*}{ Variável } & \multicolumn{2}{|c|}{ Clarity } & \multicolumn{2}{|c|}{ Damon Clear } & \multicolumn{2}{|c|}{ In-Ovation } & \multicolumn{2}{|c|}{ Quicklear } & \multirow[t]{2}{*}{$\mathbf{P}$} \\
\hline & Média & D.P. & Média & D.P. & Média & D.P. & Média & D.P. & \\
\hline $3,0 \mathrm{~mm}$ & 3907,90 & 163,41 & 3545,94 & 282,30 & 3793,61 & 315,58 & 3545,45 & 269,62 & 0,005 \\
\hline $2,0 \mathrm{~mm}$ & 2268,13 a & 552,03 & $\underset{a, b}{1762,58}$ & 489,74 & $\begin{array}{c}2013,76 \\
\mathrm{a}, \mathrm{b}\end{array}$ & 358,40 & $\underset{1790,67}{\mathrm{~b}}$ & 328,00 & $0,012^{\star}$ \\
\hline $1,0 \mathrm{~mm}$ & $1031,71 \mathrm{a}$ & 514,10 & $672,34 \mathrm{~b}$ & 322,44 & $\begin{array}{c}905,71 \\
b, \mathrm{c}\end{array}$ & 338,30 & $\begin{array}{c}994,31 \\
a, b\end{array}$ & 294,88 & $0,002^{*}$ \\
\hline $0,5 \mathrm{~mm}$ & 282,52 & 254,28 & 179,79 & 159,98 & 254,21 & 198,67 & 452,29 & 298,14 & 0,062 \\
\hline
\end{tabular}

*Estatisticamente significante para $p<0,05$.

Diferentes letras indicam a presença de diferença estatisticamente significante entre os grupos, indicados pelo Teste de Tukey.

Por fim, a tabela 4 mostra o comportamento dos braquetes quando em contato com o fio High Aesthetic. Diferentemente dos demais fios, observou-se que, para todas as deflexões estudadas, tivemos valores estatisticamente significantes, porém sem uma característica em comum, mas ainda com o braquete Clarity apresentando valores maiores, principalmente nas deflexões de 1 e $3 \mathrm{~mm}$.

Tabela 4: Média (em cN) e desvio padrão (D.P.) dos resultados do teste ANOVA a um critério de seleção e teste de Tukey, quando necessário, para avaliar o comportamento dos braquetes quando empregados com o fio High Aesthetic GAC (N=7)

\begin{tabular}{|c|c|c|c|c|c|c|c|c|c|}
\hline \multirow[t]{2}{*}{ Variável } & \multicolumn{2}{|c|}{ Clarity } & \multicolumn{2}{|c|}{ Damon Clear } & \multicolumn{2}{|c|}{ In-Ovation } & \multicolumn{2}{|c|}{ Quicklear } & \multirow[t]{2}{*}{ P } \\
\hline & Média & D.P. & Média & D.P. & Média & D.P. & Média & D.P. & \\
\hline $3,0 \mathrm{~mm}$ & $3083,31 a$ & 192,66 & $2601,92 \mathrm{~b}$ & 371,96 & $\begin{array}{c}2799,28 \\
\mathrm{a}, \mathrm{b}\end{array}$ & 159,07 & $\begin{array}{c}2885,28 \\
\mathrm{a}, \mathrm{b}\end{array}$ & 172,15 & $0,007^{*}$ \\
\hline $2,0 \mathrm{~mm}$ & $\begin{array}{c}1547,65 \\
a, c\end{array}$ & 166,24 & $1217,20 \mathrm{~b}$ & 276,00 & 1566,34 a & 46,04 & $\begin{array}{c}1305,19 \\
b, c\end{array}$ & 80,80 & $0,001^{*}$ \\
\hline $1,0 \mathrm{~mm}$ & $1466,26 \mathrm{c}$ & 385,16 & $718,78 \mathrm{~b}$ & 243,66 & $\begin{array}{c}959,06 \\
a, c\end{array}$ & 358,55 & $\begin{array}{c}1103,17 \\
\mathrm{a}\end{array}$ & 282,97 & $0,001^{*}$ \\
\hline $0,5 \mathrm{~mm}$ & $118,22 \mathrm{a}, \mathrm{c}$ & 138,87 & $83,14 \mathrm{a}, \mathrm{c}$ & 94,06 & $204,08 \mathrm{c}$ & 140,26 & $634,19 \mathrm{~b}$ & 215,61 & $0,001^{*}$ \\
\hline
\end{tabular}

*Estatisticamente significante para $p<0,05$.

Diferentes letras indicam a presença de diferença estatisticamente significante entre os grupos, indicados pelo Teste de Tukey.

\section{DISCUSSÃO}

Devido à escassez de trabalhos científicos na literatura sobre as propriedades mecânicas dos fios estéticos disponíveis no mercado, ainda persiste a dúvida se os fios metálicos recobertos possuem propriedades mecânicas semelhantes aos dos fios metálicos originais, podendo assim substituí-los e atender à exigência da estética requerida pelos pacientes. Sendo assim, no presente trabalho buscamos analisar o comportamento mecânico desses fios a fim de preencher esta lacuna no 
tocante a produção do conhecimento, pois objetiva avaliar as características de força-deflexão de fios estéticos disponíveis no mercado, utilizando-se de um modelo de simulação clínica, com diferentes tipos de braquetes autoligáveis (passivos e ativos), utilizados durante a fase inicial de alinhamento e nivelamento do tratamento ortodôntico.

Não existe um consenso na literatura sobre a quantidade de espécimes empregada para cada tipo de fio. Da Silva et al.5 utilizaram 5 amostras para cada tipo de fio, sendo que ljima et al.6 utilizaram somente uma. A norma internacional ISO 15481 indica uma amostra maior ou igual a seis espécimes para cada tipo de fio. Este e outros trabalhos7-9 utilizaram 10 amostras para cada tipo de fio estudado, como indicado na norma supracitada. É importante ressaltar que todos os fios testados nessa pesquisa foram empregados uma única vez no dispositivo de simulação clínica.

Embora o teste de deflexão elástica na máquina de três pontos seja amplamente utilizado por vários autores5-8,10 por simularem a pressão do fio aos dentes durante o tratamento ortodôntico e oferecerem reprodutibilidade, facilitando assim comparações entre estudos, nesta pesquisa utilizamos um dispositivo de simulação clínica, conforme relatam outros autores 5,8,9,11,12 que buscaram incrementar ou modificar os testes de deflexão de três pontos incluindo a estes variáveis como tipos de braquetes e ligaduras elásticas com intuito de reproduzirem melhor o ambiente clínico, onde situações como o atrito entre esses componentes podem influenciar a magnitude da força. Por outro lado, como são introduzidas muitas variáveis, a reprodutibilidade dos resultados fica mais difícil, limitando comparações com outros trabalhos6,7,11,13.

Apesar dos esforços para reproduzir uma situação clínica, cada estudo apresenta suas particularidades na avaliação das propriedades mecânicas dos fios. Por isso, sugere-se que uma maior ênfase seja atribuída à ordem da magnitude das forças produzidas pelos fios e não ao valor numérico da quantidade de força liberada pelos mesmos 14 .

Os resultados da presente pesquisa mostraram que, para todos os fios de $\mathrm{NiTi}$ com diferentes tipos de recobrimento estético estudados, as maiores forças de deflexão foram apresentadas pelo braquete Clarity (autoligável passivo) seguido pelo braquete In-Ovation (autoligável ativo). Sugere-se, assim, que quando os fios estéticos de NiTi estiverem em contato com os braquetes acima citado, menores níveis de atrito estejam agindo no sistema fio/braquete e, por consequência, a maior parte da energia armazenada no fio é capaz de retornar na forma de movimentação dentária durante a desativação.

Em virtude do que foi exposto acima, os resultados deste trabalho não permitem uma comparação profunda com outros trabalhos, pois, aspectos como diâmetro dos fios, valores de deflexão, presença ou não de braquetes e temperatura dos testes são variáveis primordiais nesse tipo de pesquisa que precisam ser levadas em consideração5,6,8,9,11,15,16.

De acordo com a norma ISO 15841 a tolerância máxima de variação da secção dos arcos deve ser de aproximadamente $0,01 \mathrm{~mm}(0,0003937$ polegadas $)$. Fios recobertos por diferentes tipos de materiais estéticos podem apresentar diâmetros diferentes do especificado pelo fabricante em sua embalagem, fator este extremamente relevante quando analisamos o comportamento mecânico desses fios, pois pode resultar tanto uma diminuição do calibre do fio de NiTi interno, como em um aumento excessivo na quantidade de material de recobrimento.

A redução nas dimensões da liga interna de $\mathrm{NiTi}$ a fim de compensar a espessura do revestimento parece ser a variável responsável pelas maiores alterações nas propriedades mecânicas dos fios estéticos, particularmente nas forças de deflexão elástica, como verificadas nesse trabalho.

Observou-se que os fios estéticos utilizados neste trabalho apresentaram em sua maioria forças de deflexão compatíveis àquelas obtidas pelos fios de NiTi. Quando fios de NiTi com diferentes tipos de cobertura estética são comparados, observa-se uma significativa diferença na intensidade das forças produzidas por eles.

Como ainda há poucos trabalhos na literatura sobre as propriedades mecânicas dos fios estéticos, sugere-se que mais estudos sejam realizados, a fim de que estas propriedades mecânicas estejam compatíveis com os níveis de força desejados na movimentação dentária induzida.

\section{CONCLUSÃO:}

De acordo com a metodologia empregada no presente trabalho, pôde-se concluir que as maiores forças de deflexão, durante a desativação, foram exercidas pelos fios Aesthetic Polymer Aditek e Flexy NiTi Rhodium em conjunto com o braquete Clarity.

Braquetes autoligáveis cerâmicos empregados jun- 
tamente com diferentes tipos de fios de estéticos podem apresentar comportamentos diferentes principalmente em virtude das características dos materiais de recobrimento empregados nos fios.

\section{AGRADECIMENTO:}

À CNPq:

O presente trabalho foi realizado com o apoio do Conselho Nacional de Desenvolvimento Científico e Tecnológico (CNPq), através de Bolsa de Iniciação Científica (IC) (Processo 154696/2019-6).

\section{REFERÊNCIAS:}

1. Quintão CCA, Brunharo IHVP. Fios ortodônticos: conhecer para otimizar a aplicação clínica. Rev Dent Press Ortod Ortop Facial. 2009;14(6):144-57.

2. Huang ZM, Gopal R, Fujihara K, et al. Fabrication of a new composite orthodontic archwire and validation by a bridging micromechanics model. Biomaterials. 2003;24(17):2941-53.

3. Sathler R, Silva RG, Janson G, Branco NCC, Zanda M. Desmistificando os braquetes autoligáveis. Dent Press J Orthod. 2011;16(2):e1-8.

4. Kusy RP. A review of contemporary archwires: their properties and characteristics. Angle Orthod. 1997;67(3):197-207.

5. Silva DL, Mattos CT, Sant'Anna EF, Ruellas ACO, Elias CN. Cross-section dimensions and mechanical properties of esthetic orthodontic coated archwires. Am J Orthod Dentofac Orthop. 2013;143(4 Suppl):S85-91.

6. lijima M, Muguruma T, Brantley W, et al. Effect of coating on properties of esthetic orthodontic nickel-titanium wires. Angle Orthod. 2012;82(2):319-25.

7. Ballard RW, Sarkar NK, Irby MC, Armbruster PC, Berzins DW. Three-point bending test comparison of fiber-reinforced composite archwires to nickel-titanium archwires. Orthodontics. 2012;13(1):46-51.

8. Elayyan F, Silikas N, Bearn D. Mechanical properties of coated superelastic archwires in conventional and self-ligating orthodontic brackets. Am J Orthod Den- tofac Orthop. 2010;137(2):213-7.

9. Kaphoor AA, Sundareswaran S. Aesthetic nickel titanium wires--how much do they deliver? Eur J Orthod. 2012;34(5):603-9.

10. Elayyan F, Silikas N, Bearn D. Ex vivo surface and mechanical properties of coated orthodontic archwires. Eur J Orthod. 2008;30(6):661-7.

11. Santoro M, Nicolay OF, Cangialosi TJ. Pseudoelasticity and thermoelasticity of nickel-titanium alloys: a clinically oriented review. Part II: Deactivation forces. Am J Orthod Dentofac Orthop 2001;119(6):594-603.

12. Burstone CJ, Liebler SAH, Goldberg AJ. Polyphenylene polymers as esthetic orthodontic archwires. Am J Orthod Dentofac Orthop. 2011;139(4 Suppl):e391-8.

13. Nakano H, Satoh K, Norris R, et al. Mechanical properties of several nickel-titanium alloy wires in three-point bending tests. Am J Orthod Dentofac Orthop. 1999;115(4):390-5.

14. Wilkinson PD, Dysart PS, Hood JAA, Herbison GP. Load-deflection characteristics of superelastic nickel-titanium orthodontic wires. Am J Orthod Dentofac Orthop. 2002;121(5):483-95.

15. Imai T, Watari F, Yamagata S, Kobayashi M, Nagayama K, Nakamura S. Effects of water immersion on mechanical properties of new esthetic orthodontic wire. Am J Orthod Dentofac Orthop. 1999;116(5):533-8.

16. Imai T, Watari F, Yamagata $S$, et al. Mechanical properties and aesthetics of FRP orthodontic wire fabricated by hot drawing. Biomaterials. 1998;19(23):2195200. 